\title{
Biomarkers in the pathogenesis, diagnosis, and treatment of psoriasis
}

\author{
This article was published in the following Dove Press journal: \\ Psoriasis: Targets and Therapy \\ 3 September 2012 \\ Number of times this article has been viewed
}

\author{
Silvia Molteni \\ Eva Reali \\ Laboratory of Translational \\ Immunology, Istituto Ortopedico \\ Galeazzi, Milan, Italy
}

Correspondence: Eva Reali Laboratory of Translational Immunology, Istituto Ortopedico Galeazzi, via Riccardo Galeazzi 4, Milan 2016I, Italy

Tel +3902 662I 4780

Fax +3902662I 4048

Email eva.reali@grupposandonato.it
Abstract: Development of psoriasis results from a complex interplay between genetically predisposing factors and environmental triggers that give rise to a self-sustaining pathogenic cycle involving $\mathrm{T}$ cells, dendritic cells, connective tissue, and skin epithelium. From $5 \%$ to $40 \%$ of patients with psoriasis also develop psoriatic arthritis, and increasing evidence indicates an association with other systemic manifestations, including cardiovascular disease and the metabolic syndrome. In psoriatic disease, there is a need for development of biomarkers for assessment of disease severity, for prediction of the outcome of therapeutic interventions, and for distinction between the different clinical variants of the disease. A field of great importance is identification of biomarkers for prediction of development of comorbidities, such as arthritis, cardiovascular disease, and the metabolic syndrome. Genetic determinants of psoriasis and their products not only give an important insight into the pathogenesis of the disease, but may also function as markers of risk for developing cutaneous psoriasis or psoriatic arthritis. So far, there are limited validation data to support the use of candidate biomarkers in clinical practice. Here we review the data from several studies on some of the most promising candidate biomarkers for cutaneous psoriasis and psoriatic arthritis, for the detection of systemic inflammation, and for use as endpoints for therapeutic interventions. Attention is focused on the molecules that take part in the interplay giving rise to psoriasis and on gene products that may represent a link between predisposing genetic factors and the immune and inflammatory processes involved in pathogenesis of the disease. Finally, we provide an overview on how biomarkers can offer insights into the pathogenesis and natural history of psoriasis.

Keywords: psoriatic disease, genetic markers, tissue-associated biomarkers, serum biomarkers, predictive factors, comorbidities

\section{Introduction}

Psoriasis is a common chronic inflammatory skin disease affecting approximately $2 \%$ of the Caucasian population. ${ }^{1,2}$ The disease appears in different clinical variants, ie, psoriasis vulgaris (accounting for $90 \%$ of all cases of psoriasis), inverse psoriasis, generalized pustular psoriasis, palmoplantar psoriasis, and guttate psoriasis, affecting children and adolescents after a $\beta$-hemolytic streptococcal infection. ${ }^{3}$ Up until now, the distinction between the various forms of psoriasis has been based on clinical features in patients, and there are limited data on biomarkers that can be used as a diagnostic tool in clinical practice.

Psoriasis has three principal histological features, ie, epidermal hyperplasia, leukocyte infiltrates, and an increased number of tortuous and leaky vessels in the dermis. Other histological characteristics are the absence of papillomatosis and an increased 
mitotic rate of basal keratinocytes, resulting in acanthosis of the epidermis and elongation of rete ridges. ${ }^{2-4}$ In the hyperproliferative epidermis of the psoriatic plaque, keratinocytes are characterized by an abnormal and incomplete differentiation process resembling the regenerative maturation observed during wound repair., ${ }^{4,5}$

Psoriasis was indeed originally considered to be a disease involving mainly keratinocytes whereas, according to the current view, the histological features of psoriasis arise as a consequence of an uncontrolled self-maintaining proinflammatory cycle in the dermis driven by cross-talk between T cells, dendritic cells, connective tissue, and skin epithelium (Figure 1). ${ }^{4,6}$ In the dermis, T cells and dendritic cells form perivascular aggregates that have been hypothesized to function as a secondary lymphoid tissue. ${ }^{6,7}$ At this level, T cell polarization occurs while activated keratinocytes and connective tissue cells secrete cytokines, growth factors, including vascular endothelial growth factor (VEGF) that promotes formation of new vessels, and chemokines that attract $T$ cells and monocytes from the circulation, thus sustaining the pathogenic cycle (Figure 1). ${ }^{2,6}$ The pivotal role of T cells in the pathogenesis of psoriasis was originally suggested by a case of psoriasis which developed after bone marrow transplantation from a donor with psoriasis and was subsequently evidenced in human skin xenografted SCID mouse models. In humans, the role of $\mathrm{T}$ cells was definitely confirmed by the clinical outcome of $\mathrm{T}$ cell-targeted therapies, such as cyclosporine A, alefacept, and efalizumab, in the treatment of psoriasis. ${ }^{8-13}$ Th1 cells were considered for a long time to be the main mediators of the immune response in psoriatic plaques, but in recent years, several studies have pointed to a critical role of Th17 cells in sustaining skin inflammation. ${ }^{14}$ Th17 cytokines, interleukin (IL)-17 A and IL-22, stimulate production of antimicrobial peptides by keratinocytes that, in turn, promote recruitment of inflammatory cells. Very recently, it has been reported that IL-17 itself is capable of enhancing keratinocyte proliferation and inhibiting keratinocyte differentiation via the downstream mediator REG3A, a protein with antimicrobial functions involved in wound repair. ${ }^{15}$ IL-22 is also capable of stimulating keratinocyte proliferation via induction of IL-20. ${ }^{2,16}$

Genetic susceptibility factors strongly contribute to the predisposition to psoriasis. Population studies have shown that the incidence of psoriasis is higher among people who have affected relatives, and twin studies have shown that monozygotic twins have a 2-3 times higher risk of developing psoriasis than dizygotic twins. ${ }^{3,17}$ Gene variants in psoriasis have been well described by linkage analyses and genome-wide association scans. Linkage analysis studies have identified nine chromosomal loci associated with psoriasis named PSORS1 to PSORS9. ${ }^{5,18}$ The major genetic determinant of psoriasis resides in the locus of susceptibility, PSORS1, encoding the gene variant HLA-Cw6, which is carried by up to $60 \%$ of patients with early-onset psoriasis. ${ }^{3,19}$ Another locus of susceptibility associated with epidermal differentiation pathways is the epidermal differentiation

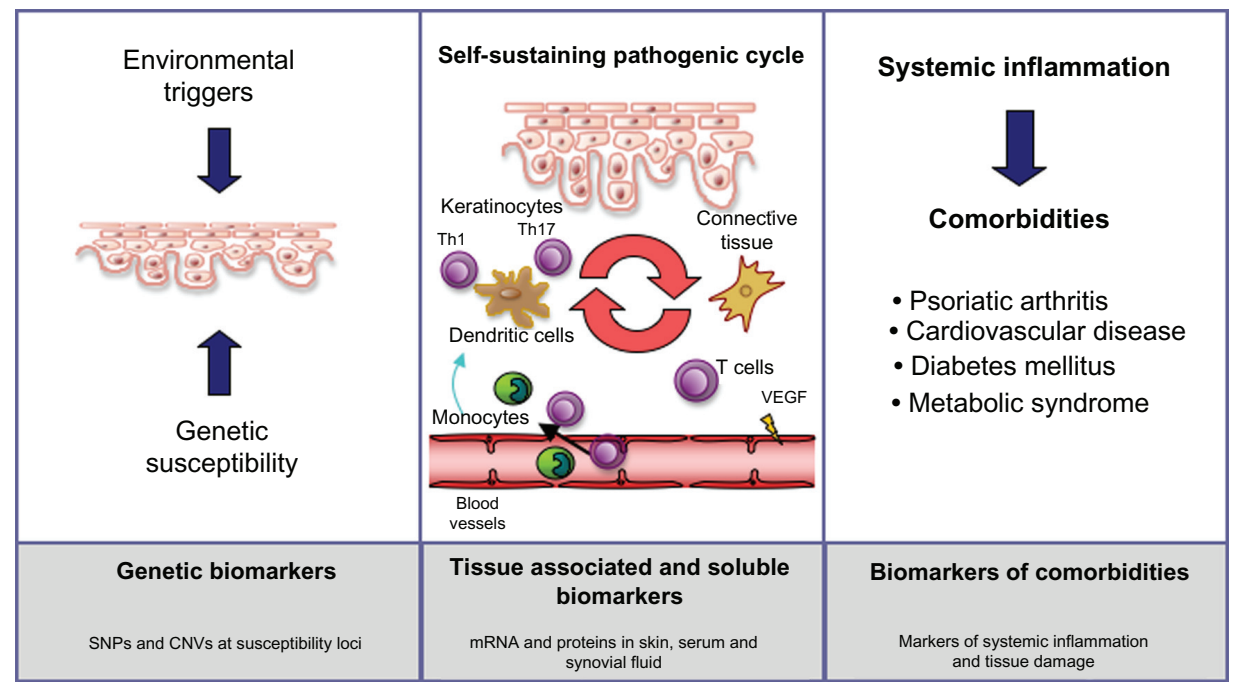

Figure I Psoriasis development: from genetic to comorbidities.

Notes: Schematic representation of the development of psoriatic disease and of the self-sustaining pathogenic cycle in the derma. Skin plaque formation is induced by environmental triggers in genetic predisposed subjects. Maintenance of the plaque is mediated by a self-sustaining pathogenic cycle constituted by $T$ cells, dendritic cells, connective tissue (fibroblasts) and skin epithelium (keratinocytes). Moreover, the increased vascularity of the derma allows the migration from the circulation into the inflamed tissue of $\mathrm{T}$ cells and monocytes that will differentiate into mature dendritic cells. The systemic inflammatory condition characterizing psoriasis is evidenced by the high circulating levels of inflammation markers and, in a fraction of patients, causes the development of comorbidities, such as psoriatic arthritis and cardiovascular diseases. Abbreviations: SNP, single-nucleotide polymorphism; CNV, copy number variation; VEGF, vascular endothelial growth factor. 
complex gene cluster (PSORS4), that is likely to be involved in terminal differentiation of keratinocytes. PSORS4 contains variations in copy numbers of genes belonging to the late cornified envelope complex and also contains genes encoding the S100 calcium-binding proteins that are produced by keratinocytes and have proinflammatory and chemotactic functions. Other gene variants associated with psoriasis, mostly single nucleotide polymorphisms, belong to the Th1/ Th17 pathway (Il23R, Il12B, Il23A genes) and the NF- $\mathrm{BB}$ pathway (TNFAIP3). At the locus encoding human beta defensins, there are increased copy numbers of the genes encoding human beta defensin-2 (DEFB4), human beta defensin-3, and human beta defensin-4 associated with psoriasis. $^{5,18}$ Single nucleotide polymorphisms associated with psoriasis have also been detected at the tumor necrosis factor alpha (TNF- $\alpha$ ) gene promoter, and meta-analysis studies have evidenced an association between the $-238 \mathrm{G} / \mathrm{A}$ single nucleotide polymorphism and psoriasis. Importantly, different single nucleotide polymorphisms at the promoter of the gene encoding TNF- $\alpha$ have been reported to be differentially associated with cutaneous psoriasis and psoriatic arthritis. In particular, the $-857 \mathrm{C} / \mathrm{T}$ single nucleotide polymorphism has been reported to be more associated with psoriatic arthritis, and may represent a marker of risk for psoriatic arthritis. ${ }^{20,21}$ Genetic susceptibility loci of psoriasis not only give important insights into the pathogenesis of the disease but may also function as markers of risk of developing cutaneous psoriasis or psoriatic arthritis.

Recently, there has been increasing awareness that psoriasis is more than skin deep, and that the systemic inflammatory condition associated with the disease can be responsible for comorbidities, including diabetes mellitus, metabolic syndrome, and cardiovascular disease. Importantly, 5\%-40\% of affected patients develop psoriatic arthritis., ${ }^{2,3,22-26}$ The diagnosis of psoriatic arthritis is difficult because it has heterogeneous manifestations, and clinical studies suggest that there is a high prevalence of undiagnosed psoriatic arthritis in patients with psoriasis. ${ }^{27-29}$ Therefore, the identification and validation of biomarkers of psoriatic arthritis is a high priority in the field of psoriasis and psoriatic arthritis research.

According to the National Institutes of Health Biomarkers Definitions Working Group, a biomarker is "a characteristic that is objectively measured and evaluated as an indicator of normal biological processes, pathogenic processes, or pharmacologic responses to a therapeutic intervention". Biomarkers can be divided into three categories: type 0 biomarkers, that correlate longitudinally with the severity of the disease; type I biomarkers, that reflect the effect of an intervention according to the mechanism of action of the therapy itself; and type II biomarkers, that are surrogate endpoints for a therapy. ${ }^{30}$ They can be represented by soluble, cellular, tissue-associated, or genetic markers.

In psoriatic disease, biomarkers could be relevant for distinction between the different clinical variants of the disease, for assessment of disease activity and severity, and for prediction of the outcome of a therapeutic intervention. Biomarkers could also allow the selection of patient-tailored therapy to maximize the beneficial effect.

A field of great importance is the use of biomarkers for prediction of development of comorbidities such as arthritis, cardiovascular disease, and metabolic syndrome. Finally, biomarkers can also provide insights into the mechanisms involved in the pathogenesis of the disease. So far, there are limited validation data to support the use of candidate biomarkers in clinical practice for diagnosis, assessment of disease activity, or the prognosis of psoriatic disease.

The major concern for validation is the lack of quality control and insufficient statistical analysis, so there is a need for standards to develop validation criteria for biomarker selection. Here we review the data from several studies on some of the most valuable candidate biomarkers for cutaneous psoriasis and psoriatic arthritis, detection of systemic inflammation, and assessment of the therapeutic outcome. We focus our attention on the molecules that take part in the complex interplay giving rise to psoriasis and on the gene products that may represent a link between predisposing genetic factors and the immune and inflammatory processes involved in the pathogenesis of the disease (Figure 1). Finally, we provide an overview on how biomarkers can provide insights into the pathogenesis of psoriasis.

\section{Biomarkers of cutaneous psoriasis}

The current gold standard for assessment of disease activity has been the Psoriasis Area and Severity Index (PASI). The limitations of using the PASI score in clinical practice and clinical research are related to the low reproducibility and high variability of the PASI score between physicians. ${ }^{31,32}$ A quantitative way of measuring the severity of psoriasis is by evaluation of histological changes in skin biopsies. Keratins can be used as biomarkers of psoriasis severity because immunostaining with anti-K16, anti-K6, anti-K1, and anti-K10 antibodies reflects abnormal hyperproliferation and differentiation of keratinocytes in the psoriatic epidermis (Table 1). ${ }^{33-35} \mathrm{~K} 16$ is upregulated in the suprabasal layers of the interfollicular epidermis, and effective treatment leads to downregulation of K16. ${ }^{33}$ It has also been shown 
Table I Biomarkers of psoriatic disease

\begin{tabular}{|c|c|c|c|c|}
\hline Proposed biomarkers & Name/function & Association & Type & Ref \\
\hline \multicolumn{5}{|c|}{ Tissue associated biomarkers } \\
\hline $\mathrm{KI}, \mathrm{K} 6, \mathrm{KIO}, \mathrm{KI} 6$ & Keratins & Psoriasis & 0 & $30-32$ \\
\hline VEGF & Vascular endothelial growth factor & Psoriasis & 0,1 & $4 I$ \\
\hline SI00A8/A9 & SI00 calcium-binding proteins & Psoriasis & 0 & 48,49 \\
\hline IL-6 & Proinflammatory cytokines & Psoriasis & 0 & 63 \\
\hline IL-8 & & Psoriasis & 0 & 61,62 \\
\hline IL-I8 & & Psoriasis & 0 & 66 \\
\hline TNF- $\alpha$ & & Psoriasis & 0 & 63 \\
\hline IFN- $\gamma$ & ThI and ThI7 cytokines & Psoriasis & 0,1 & 63 \\
\hline IL-I7 & & Psoriasis & $0,1,2$ & 63 \\
\hline IL-22 & & Psoriasis & 0 & 63 \\
\hline TLR4 & Toll-like receptor 4 & Guttate psoriasis & na & 68 \\
\hline $\begin{array}{l}\text { Oxidative stress markers } \\
\text { (eg, oxidised LDL) }\end{array}$ & Lipid peroxidation & Psoriasis/PsA & 0 & 32 \\
\hline \multicolumn{5}{|l|}{ Soluble biomarkers } \\
\hline \multicolumn{5}{|l|}{ Serum/plasma } \\
\hline CRP & C-reactive protein, acute-phase reactant & Psoriasis/PsA & 0,2 & $35,36,69-7 \mid$ \\
\hline ESR & Erytrocyte sedimentation rate & Psoriasis/PsA & 0,2 & 40 \\
\hline VEGF & Vascular endothelial growth factor & Psoriasis/PsA & $0,1,2$ & 42,43 \\
\hline hBD-2 & $\beta$-defensin-2 & Psoraisis & 0 & 15,47 \\
\hline SI00A8/A9 & SI00 calcium-binding proteins & Psoriasis & 0 & 54 \\
\hline IL-6 & Proinflammatory and chemotactic cytokine & Psoriasis/PsA & 0 & $56,63-65$ \\
\hline IL-8 & & Psoriasis & 0 & $56,63,64$ \\
\hline IL-I8 & & Psoriasis & 0 & $56,63,66,67$ \\
\hline TNF- $\alpha$ & & Psoriasis & 0 & 56,63 \\
\hline IFN- $\gamma$ & ThI/ThI7 cytokines & Psoriasis & 0,1 & 56,64 \\
\hline IL-I7 & & Psoriasis & $0,1,2$ & 50,56 \\
\hline IL-22 & & Psoriasis & 0 & 50 \\
\hline TGF- $\beta$ I & Transforming growth factor- $\beta$ I & Psoriasis & 0 & 67 \\
\hline Leptin & Adipokines, proinflammatory functions & Psoriasis & 0 & $91-93$ \\
\hline Resistin & & Psoriasis & 0 & 91,93 \\
\hline TIMP-I & Tissue inhibitor of metalloproteinase-I & PsA & 0 & 67 \\
\hline MMP-I & Metalloproteinase-I & PsA & 0 & 67 \\
\hline CPII:C2C ratio & $\begin{array}{l}\text { C-propeptide of type II collagen:collagen } \\
\text { fragment neoepitopes ratio }\end{array}$ & PsA & 0 & 77 \\
\hline MMP-3 & Metalloproteinase-3 & PsA & 0 & 77 \\
\hline OPG & Osteoprotegerin & PsA & 0 & 77 \\
\hline \multicolumn{5}{|l|}{ Synovial fluid } \\
\hline IL-I $\beta$ & Proinflammatory cytokines & PsA & 0 & 79 \\
\hline IL-6 & & PsA & 0 & 79 \\
\hline IL-22 & & PsA & 0 & 79 \\
\hline \multicolumn{5}{|l|}{ Cell subsets } \\
\hline Thl & T helper subsets & Psoriasis & 0 & 57 \\
\hline ThI7 & & Psoriasis & 0 & 57 \\
\hline Th22 & & Psoriasis & 0 & 57 \\
\hline NKT cells & Natural killer T cells & Psoriasis & 0 & \\
\hline Osteoclast precursors & Bone erosion marker & PsA & 0 & 81,82 \\
\hline \multicolumn{5}{|l|}{ Genetic markers } \\
\hline PSORS4 SI00 & SI00 calcium-binding proteins & Psoriasis & na & 5,15 \\
\hline CNV DEFB4 & $\beta$-defensin-2 & Psoriasis & na & 5,15 \\
\hline SNPs $I 123 r$ & Proinflammatroy cytokines & Psoriasis & na & 5,15 \\
\hline SNPs $I / I 2 b$ & & PsA & na & 5,15 \\
\hline SNPs $1123 a$ & & Psoriasis & na & 5,15 \\
\hline $\begin{array}{l}\text { SNPs TNF- } \alpha \text { encoding } \\
\text { gene promoter }\end{array}$ & & Psoriasis/PsA & na & 17,18 \\
\hline NKG2D & $\begin{array}{l}\text { Activating KIR } \\
\text { (Killer cell Immunoglobulin-like receptor) }\end{array}$ & PsA & na & 84,85 \\
\hline
\end{tabular}

Abbreviation: na, not applicable; SNP, single-nucleotide; IL, interleukin; PSA, psoriatic arthritis. 
that induction of K16 in relapsing psoriasis occurs at early stages during development of a plaque, ${ }^{36}$ and increased K16 expression in nonlesional psoriatic epidermis has been suggested as a marker of preclinical psoriasis. ${ }^{37}$ Other keratin types, such as K1 and K10, representing markers of terminal differentiation of keratinocytes are instead downregulated in psoriatic skin lesions. ${ }^{33}$

With regard to serum biomarkers, the inflammatory condition of psoriasis is confirmed by higher levels of C-reactive protein detected in cross-sectional studies of patients with psoriasis compared with controls. Importantly, a significant positive correlation has been found between C-reactive protein levels and disease severity. ${ }^{38,39}$ Although in the past discordant findings have been reported for the correlation between C-reactive protein levels and disease activity in patients with psoriatic disease, the development of a highly sensitive $\mathrm{C}$-reactive protein method for detection of concentrations $<5 \mathrm{mg} / \mathrm{L}$ allowed reliable results to be obtained on levels of circulating C-reactive protein and their correlation with the PASI score. ${ }^{38,40-42}$ Other systemic inflammatory markers which are raised in patients with psoriasis compared with healthy subjects include the erythrocyte sedimentation rate and fibrinogen levels. ${ }^{43}$

A very promising candidate molecule which could serve as a soluble or tissue-associated biomarker of psoriasis is VEGF. Overexpression of VEGF in the pathogenesis of psoriasis is related to increased vascularity in the dermis, which is one of the distinctive features of psoriatic plaques. . $^{2,3,6,44}$ VEGF expression is increased in skin lesions at both the protein and mRNA levels compared with uninvolved skin and normal control skin. Importantly, mRNA expression levels were found to correlate with PASI score. ${ }^{44}$ In the serum of patients with severe forms of psoriasis, VEGF levels were found to be increased and to correlate with disease severity. ${ }^{45}$ Indeed, circulating levels of VEGF were higher during active psoriasis and in the presence of psoriatic arthritis, and were lowered during disease remission. ${ }^{46}$ Because of this evidence and because of its role in the pathogenesis of the disease, VEGF could represent a promising candidate molecule leading to the development of a biomarker for active psoriasis and psoriatic arthritis, and could represent a factor predictive of disease progression.

Another molecule that has been proposed as a candidate biomarker for psoriasis is human beta defensin-2, an antimicrobial peptide expressed at higher levels in psoriatic plaques. ${ }^{47-49}$ High human beta defensin-2 expression levels can be related to a gene effect arising from copy number variation in DEFB4 encoding the human beta defensin-2 that is associated with psoriasis. Circulating levels of human beta defensin-2 were also found to be increased in patients with psoriasis compared with controls, and to correlate with the PASI score. This recent evidence makes human beta defensin- 2 a candidate biomarker of disease activity that can be measured both in the circulation and in tissue. $5,18,50$

S100A7 (psoriasin), S100A8 (calgranulin A), S100A9 (calgranulin B), and S100 calcium-binding proteins with proinflammatory and chemotactic activity and encoded by the epidermal differentiation complex gene cluster (PSORS4) have been found to be markedly upregulated in psoriatic plaques. ${ }^{51,52}$ In psoriatic skin, expression of S100A7, S100A8, and S100A9 is induced by IL-22 and leads to proliferation of keratinocytes and production of antimicrobial peptides. ${ }^{16,53-56}$ Higher circulating levels of S100A8 and S100A9 were found in patients with psoriasis compared with controls, and their concentration in serum was found to correlate positively with disease activity. ${ }^{57,58}$

Cytokines involved in the pathogenesis of psoriasis have also been proposed as soluble biomarkers of the disease. In particular, increased serum concentrations of IL-17 and interferon gamma have been reported to correlate with the PASI score. ${ }^{53,59,60}$ Furthermore, increased levels of the p40 subunit, common to IL-12 and IL-23, have been reported in lesional skin compared with normal skin, and IL-20, the IL-22 induced cytokine that promotes hyperproliferation of keratinocytes, was also found to be present at higher levels in psoriatic skin. ${ }^{61-63}$

Among the inflammatory cytokines, IL-6 and IL-8 have been shown to be increased in both the circulation and in lesional skin compared with controls. ${ }^{64-68}$ The candidate cytokine biomarker of disease severity supported by the greatest amount of evidence is IL-18. Different groups have reported that IL-18 expression is higher in active plaques than in established lesions, is characterized by intensive inflammation and desquamation, and its serum levels are significantly higher in patients with psoriasis than in controls and are positively correlated with the PASI score. ${ }^{59,69,70}$

Many other molecules have been proposed as biomarkers of cutaneous psoriasis, including metalloproteinase-1 (a marker of tissue damage), transforming growth factor- $\beta 1$, and tissue inhibitor of metalloproteinase- $1 .{ }^{70}$ Intriguingly, increased expression of Toll-like receptor 4 has been found in guttate psoriasis, suggesting that Toll-like receptor 4 may be related to the pathogenesis of this clinical variant, which arises after bacterial infection. ${ }^{71}$

Finally, regarding cell subsets that could represent biomarkers of psoriasis, Th17, Th1, and Th22 cells have 
been suggested. Recently, we have also reported that patients with cutaneous psoriasis have higher percentages of circulating natural killer $\mathrm{T}$ cells when compared with controls and patients with psoriatic arthritis (Bosè et al, unpublished data, Table 1). Although detection of different percentages of these cell subsets in the circulation can provide important insights into the pathogenesis of the disease, no conclusive evidence has been provided in support of their possible use as biomarkers.

All the proposed biomarkers are related to the pathogenic mechanisms of psoriasis, so represent valuable tools to assess disease severity and the outcome of a therapeutic intervention. However, they can be considered as additional measures to be used in parallel with the PASI score. Finding a correlation between cellular and/or molecular markers and the PASI score will lead to a very comprehensive understanding of the molecular mechanisms underlying the clinical features of psoriasis.

\section{Biomarkers for psoriatic arthritis}

The identification of biomarkers capable of predicting the development of psoriatic arthritis in patients with psoriasis represents a high priority in this field. Recently, an international goal-directed study to determine biomarkers for joint damage in psoriatic arthritis has been initiated through a collaboration between the GRAPPA (the Group for Research and Assessment of Psoriasis and Psoriatic Arthritis) and OMERACT (Outcome Measures for Rheumatology Clinical Trials) groups.

Clinical studies have indicated a high prevalence of undiagnosed psoriatic arthritis in patients with psoriasis. Indeed, diagnosis of psoriatic arthritis is more difficult compared with other forms of arthropathy because of the lack of existing systemic biomarkers for rheumatoid arthritis. The diagnostic criteria used for psoriatic arthritis include the Moll and Wright and CASPAR classification methods. The Moll and Wright system defines psoriatic arthritis as an inflammatory arthritis involving distal interphalangeal joints, with symmetric polyarthritis and oligoarticular arthritis, predominant axial spondylitis, and arthritis mutilans. ${ }^{72}$ The CASPAR system is based on both clinical and genetic criteria, considering the family history of psoriasis, involvement of distal joints, juxtaarticular new bone formation, nail involvement, and absence of the rheumatoid factor. ${ }^{73}$ Identification of biomarkers for psoriatic arthritis could improve these diagnostic criteria, providing quantitative information about the severity of the disease and facilitating its diagnosis, given the heterogeneity of manifestations of psoriatic arthritis.
Patients with psoriatic arthritis have an additional burden of systemic inflammation compared with patients with the cutaneous form of psoriasis, as reflected by the higher circulating levels of C-reactive protein. ${ }^{74-76} \mathrm{C}$-reactive protein levels have been found to correlate with a higher number of involved joints and to represent a negative prognostic predictor, especially for forms of psoriatic arthritis involving the peripheral joints. ${ }^{77,78}$ Another biomarker of inflammation that is increased in patients with psoriatic arthritis is the erythrocyte sedimentation rate, which also correlates with the number of affected joints. ${ }^{79}$ Biomarkers of psoriatic arthritis can be detected at the systemic level, in the synovial fluid of the affected joints, or can be represented by predisposing genetic factors. Finally, clinical features of psoriatic arthritis can serve as markers of disease severity.

At the systemic level, there are no conclusive studies focusing on the differences between patients with psoriatic arthritis and those with cutaneous psoriasis. However, in a recent study, it has been shown that patients with psoriatic arthritis have higher levels of IL-6. Serum IL-6 is proposed to represent a more specific marker than $\mathrm{C}$-reactive protein and erythrocyte sedimentation rate in patients with psoriatic arthritis, and its level was found to correlate with the number of joints affected by arthritis. ${ }^{80,81}$ Patients with psoriatic arthritis also have higher serum levels of IL-1 receptor antagonist that correlate with the number of tender and swollen joints. $^{79}$

Recently, we have reported higher levels of IL-2 secretion from circulating peripheral blood mononuclear cells upon stimulation in patients with psoriatic arthritis than in those with the cutaneous form of psoriasis and controls, that could reflect differences between the two types of patients with regard to systemic $\mathrm{T}$ cell responses (Bosè et al, unpublished data).

Other markers that have been detected in the circulation of patients with psoriatic arthritis reflect cartilage destruction and bone remodeling. These include metalloproteinase-3, osteoprotegerin, and the ratio between C-propeptide of type II collagen and collagen fragment neoepitopes (CPII:C2C). ${ }^{82}$ The serum level of the receptor activator of nuclear factor $\kappa \mathrm{B}$ ligand (RANKL) reflects the extent of bone erosion and has been proposed as a predictive marker of progressive joint damage. ${ }^{83}$ Circulating osteoclast precursors in patients with psoriatic arthritis have also been proposed as cellular biomarkers of disease severity because of their correlation with bone erosion. ${ }^{84,85}$

Other sources of biomarkers in psoriatic arthritis are the synovium and synovial fluid of affected joints. Studies have 
reported increased expression of TNF- $\alpha$, interferon gamma, IL-6, and IL-1 $\beta$ in the synovium from joints with psoriatic arthritis. ${ }^{86,87}$ In a longitudinal study of patients with psoriatic arthritis undergoing treatment with etanercept, it has been shown that basal IL-1 $\beta$, IL-6, and IL-22 levels in synovial fluid were correlated with C-reactive protein levels and these cytokines were significantly reduced after this therapy. ${ }^{86}$

The association between psoriatic arthritis and natural killer cell pathways is of increasing interest, after the presence of some allotypes of the killer cell immunoglobulin-like receptor (KIR) superfamily were found recently to influence susceptibility to psoriatic arthritis. Indeed, it has been suggested that KIR expression in the synovial fluid of patients with psoriatic arthritis is dominated by activating KIRs (NKG2D, Table 1). ${ }^{88,89}$

From the genetic point of view, patients with the cutaneous form of psoriasis and those with psoriatic arthritis share the majority of the predisposing gene variants, in particular an association with HLA-Cw6. Other class I antigens are also associated with psoriatic arthritis, including HLAB13, HLA-B57, HLA-B39, and HLA-Cw7. From linkage analysis studies, only one locus, ie, PSORAS1 (PSORS8), seems to be specifically associated with psoriatic arthritis. Genome-wide association studies have also shown that single nucleotide polymorphisms at the gene encoding IL-12B were more associated with psoriatic arthritis than with psoriasis alone. Recently, it has also been found that single nucleotide polymorphisms in the IL-13 gene region represent a possible specific risk factor for psoriatic arthritis. This hypothesis is strengthened by previous reports showing localized production of IL-13 in inflamed joints. . $^{5,18,90-93}$

Finally, from the clinical point of view, psoriatic arthritis is frequently associated with nail disease, and it has been reported that the psoriasis nail severity score is correlated with involvement of the distal interphalangeal joints, as well as with progressive and unremitting arthritis. For this reason, nail changes and especially onycholysis have been proposed as a clinical prognostic marker of psoriatic arthritis. ${ }^{94,95}$

\section{Biomarkers of comorbidities}

Psoriasis, and especially psoriatic arthritis, are associated with systemic inflammatory conditions that include type II diabetes mellitus, metabolic syndrome, and cardiovascular disease..$^{2,3,96-98}$ For this reason, it is of great importance to identify biomarkers that can predict the risk of developing comorbidities. One of the most reliable predictive markers is C-reactive protein, which is a validated biomarker of cardiovascular disease..$^{41,99}$
Another emerging comorbidity associated with psoriasis is obesity. In obese subjects, activated white adipocytes secrete proinflammatory cytokines, such as TNF- $\alpha$, and adipocytokines, such as leptin, and peripheral blood mononuclear cells secrete resistin that promotes insulin resistance and inflammation. ${ }^{100}$ In a cross-sectional study of 39 patients with moderate-to-severe psoriasis, it has been reported that serum levels of resistin are higher and correlated with PASI score. This finding was confirmed by another study of 30 obese patients with psoriasis in whom serum levels of resistin were found to be significantly higher than in controls and to correlate with disease severity. ${ }^{101,102}$ While no differences were found in circulating leptin levels in this study, another study of 43 patients reported that the leptin concentration in serum was significantly higher in those with severe psoriasis than in patients with mild-moderate psoriasis. ${ }^{103}$ Therefore, leptin and resistin could be investigated further as candidate biomarkers for prediction of development of insulin resistance and atherosclerosis in patients with psoriatic disease.

Patients with psoriatic disease also exhibit increased circulating markers of oxidative stress (eg, catalase and antioxidized low-density lipoprotein antibody) and higher neutrophil function compared with control subjects. ${ }^{35}$ Oxidative damage and lipid peroxidation may further account for atherosclerosis in psoriasis, and longitudinal studies may identify candidate oxidative stress markers as predictors of development of cardiovascular disease (Table 1).

\section{Biomarkers of clinical response to biological therapies}

Biological therapies for the treatment of psoriasis include $\mathrm{T}$ cell agents (alefacept and efalizumab), TNF- $\alpha$ inhibitors (etanercept, infliximab, adalimumab), the anti-p40 agent, ustekinumab, and more recently, the anti-IL-17 agents, ixekizumab and brodalumab. ${ }^{104-106}$

Some of the biomarkers of disease activity summarized in this review (Table 1) have been shown to decrease upon treatment with biological therapeutics and could be validated as clinical or surrogate endpoints. However, at present, reduction in PASI score (PASI75, PASI90, PASI100) remains the current endpoint for assessment of the clinical efficacy of a treatment. ${ }^{104,106}$

The most widely used soluble serum biomarker for the detection of the clinical response to biological therapies is C-reactive protein. In two randomized studies of patients with psoriatic disease treated with etanercept, it has been shown that C-reactive protein levels decreased both in patients with cutaneous psoriasis and in patients with psoriatic disease. 
In these cases, reduction of C-reactive protein correlated with disease resolution after treatment. ${ }^{42,107}$ Similar results were obtained for patients treated with infliximab or adalimumab. ${ }^{78,108,109}$ The placebo-controlled Phase III PHOENIX-1study of the efficacy of ustekinumab also demonstrated a reduction in C-reactive protein levels in patients treated with the anti-p40 antibody, reflecting amelioration of the disease. ${ }^{110}$

Reduction of C-reactive protein in patients with psoriasis upon treatment has recently been proposed as a factor predictive of reduced risk of developing cardiovascular disease. In a longitudinal study of 42 patients with severe psoriasis treated using systemic therapies (fumaric acid esters, cyclosporine A, methotrexate, etanercept, adalimumab, or ustekinumab) serum markers, such as C-reactive protein, VEGF, and resistin, were reduced by treatment in parallel with PASI score reduction that correlated significantly with downregulation of resistin. ${ }^{111}$

In a recent study, we have shown that early downregulation of Vegf expression in psoriatic plaques after anti-TNF treatment correlates strongly with the clinical response (Bosè et al, unpublished data). These findings, together with evidence of an important role for angiogenesis in the pathogenesis of the disease, make VEGF in both psoriatic plaques and serum a promising candidate marker of disease severity and response to therapies.

Gene expression changes in lesional skin can also be used effectively as biomarkers of clinical response. In particular, downregulation of Th17 pathway genes was found to discriminate between responders and nonresponders to etanercept after 12 weeks of treatment, as reported by Zaba et al. ${ }^{112}$ We have also shown that the clinical response after 12 weeks of treatment with anti-TNF agents is associated with early downregulation of the CCR7/CCL19 axis, dendritic cell maturation genes, and Vegf, suggesting that these genes could serve as an early marker of clinical response (Bosè et al, unpublished data).

It would be of great interest to detect biomarkers that can predict the clinical outcome of a treatment. These could be represented by differentially expressed genes in skin lesions that can be predictive of the beneficial effects of a given biologic agent. Recently, expression of 23 genes at the skin level has been proposed as a disease response classifier to predict the response to alefacept. Data were obtained from microarray analysis of peripheral blood mononuclear cells from 16 patients with moderate-to-severe psoriasis. ${ }^{113}$ Larger longitudinal studies will be needed, but this approach may represent a promising tool to select patients for alefacept treatment.
Recently, a whole-genome transcriptome profiling based on microarray data from 62 lesional skin samples was used to characterize the inflammatory and cytokine network in psoriatic plaques. Patients were stratified according to the inflammatory gene expression into three classes, ie, strong, moderate, or weak, and were further divided into two subgroups based on high or low expression of cytokine-specific transcriptomes (IL-13 strong or IL-13 weak). Such interpatient variability could possibly account for different treatment responses and further studies could support the use of these microarray data to predict patient responses to systemic therapies and to develop personalized treatments. ${ }^{114}$

\section{How biomarkers provide insights into pathogenesis}

Biomarkers of disease severity and clinical response to biological therapies can be very important for improvement of the clinical and therapeutic management of psoriasis. In addition to that, molecular, cellular, and genetic diseaseassociated markers can also provide important insights into the pathogenesis of the disease. The finding that downregulation of Th17 pathway genes can discriminate between responders and nonresponders to etanercept has supported the role of Th17 cells in the pathogenesis of the disease. ${ }^{112}$ In target tissues, therapeutic blockade of TNF induces modulation of expression of a wide panel of genes that reflect changes in the cell and cytokine network. According to the current view, disease remission is likely to depend mainly on modulation of the pathways that contribute most to the pathogenesis of the disease. ${ }^{112,115,116}$ On this basis, we found that downregulation of the CCR7/CCL19 axis is strongly correlated with clinical remission in anti-TNF treated patients (Bosè et al, unpublished data) pointing out an important role for the CCR7/CCL19 axis in the pathogenesis of psoriasis.

The differential expression of cytokines between patients with cutaneous psoriasis and those with psoriatic arthritis may indicate features which can distinguish between the two forms of the disease, and evaluation of the cytokine expression profile by anti-CD3-stimulated T cells may demonstrate different roles of systemic $\mathrm{T}$ cell responses in cutaneous psoriasis and psoriatic arthritis.

The genetic associations of psoriasis can also give further information about the pathogenic mechanisms of psoriatic disease and provide new biomarkers. One example is represented by copy number variations of the genes encoding human beta defensins, as reflected in the altered expression pattern of these molecules, that could be used as a biomarker of psoriasis severity. Also, identification of an association 
between activating KIR allotypes and psoriatic disease suggests a functional role for abnormal natural killer cells in the pathogenesis of psoriatic disease ${ }^{88,89}$ and could also help to identify novel targets for future therapeutic interventions.

\section{Conclusion}

Biomarkers have the potential to improve the evaluation and management of psoriatic disease. There are still limited data available to validate candidate biomarkers in the different clinical forms of the disease. Longitudinal studies in larger cohorts of patients will help to strengthen the rationale for their use in clinical practice. Promising candidate biomarkers for psoriasis include VEGF, beta defensins, and S100 proteins that can be found both in serum and in psoriatic plaques, and could be used in clinical practice to assess disease severity or as endpoints in studies of therapeutic interventions. A promising field is also the use of genetic variants as predictive risk factors for development of psoriatic disease. Moreover, detection of changes in gene expression that correlate with response to biological therapies is a promising field for the development of new biomarkers that will help to select the most effective therapy for individual patients.

\section{Acknowledgment}

We thank Professors Sergio Ottolenghi, Antonella Ronchi, Alessandra Polissi, and Silvia Barabino from the University of Milano-Bicocca, Milan, Italy, for their critical reading of the manuscript.

\section{Disclosure}

The author reports no conflicts of interest in this work.

\section{References}

1. Christophers E. Psoriasis - epidemiology and clinical spectrum. Clin Exp Dermatol. 2001;26(4):314-320.

2. Nestle FO, Kaplan DH, Barker J. Psoriasis. N Engl J Med. 2009;361(5):496-509.

3. Griffiths CE, Barker JN. Pathogenesis and clinical features of psoriasis. Lancet. 2007;370(9583):263-271.

4. Lowes MA, Bowcock AM, Krueger JG. Pathogenesis and therapy of psoriasis. Nature. 2007;445(7130):866-873.

5. Liu Y, Krueger JG, Bowcock AM. Psoriasis: genetic associations and immune system changes. Genes Immun. 2007;8(1):1-12.

6. Bowcock AM, Krueger JG. Getting under the skin: the immunogenetics of psoriasis. Nat Rev Immunol. 2005;5(9):699-711.

7. Mitsui H, Suarez-Farinas M, Belkin DA, et al. Combined use of laser capture microdissection and cDNA microarray analysis identifies locally expressed disease-related genes in focal regions of psoriasis vulgaris skin lesions. J Invest Dermatol. 2012;132(6):1615-1626.

8. Nickoloff BJ, Bonish B, Huang BB, Porcelli SA. Characterization of a $T$ cell line bearing natural killer receptors and capable of creating psoriasis in a SCID mouse model system. J Dermatol Sci. 2000;24(3):212-225.

9. Nickoloff BJ, Schroder JM, von den Driesch P, et al. Is psoriasis a T-cell disease? Exp Dermatol. 2000;9(5):359-375.
10. Krueger GG, Callis KP. Development and use of alefacept to treat psoriasis. J Am Acad Dermatol. 2003;49(2 Suppl):S87-S97.

11. Lebwohl M, Tyring SK, Hamilton TK, et al; Efalizumab Study Group. A novel targeted T-cell modulator, efalizumab, for plaque psoriasis. N Engl J Med. 2003;349(21):2004-2013.

12. Mueller W, Herrmann B. Cyclosporin A for psoriasis. N Engl J Med. 1979;301(10):555.

13. Gardembas-Pain M, Ifrah N, Foussard C, Boasson M, Saint Andre JP, Verret JL. Psoriasis after allogeneic bone marrow transplantation. Arch Dermatol. 1990;126(11):1523.

14. Di Cesare A, Di Meglio P, Nestle FO. The IL-23/Th17 axis in the immunopathogenesis of psoriasis. J Invest Dermatol. 2009;129(6): $1339-1350$

15. Lai Y, Li D, Li C, et al. The antimicrobial protein REG3A regulates keratinocyte proliferation and differentiation after skin injury. Immunity. 2012;37(1):74-84.

16. Wolk K, Witte E, Wallace E, et al. IL-22 regulates the expression of genes responsible for antimicrobial defense, cellular differentiation, and mobility in keratinocytes: a potential role in psoriasis. Eur J Immunol. 2006;36(5):1309-1323.

17. Farber EM, Nall ML, Watson W. Natural history of psoriasis in 61 twin pairs. Arch Dermatol. 1974;109(2):207-211.

18. Duffin KC, Woodcock J, Krueger GG. Genetic variations associated with psoriasis and psoriatic arthritis found by genome-wide association. Dermatol Ther. 2010;23(2):101-113.

19. Trembath RC, Clough RL, Rosbotham JL, et al. Identification of a major susceptibility locus on chromosome $6 \mathrm{p}$ and evidence for further disease loci revealed by a two stage genome-wide search in psoriasis. Hum Mol Genet. 1997;6(5):813-820.

20. Hohler T, Kruger A, Schneider PM, et al. A TNF-alpha promoter polymorphism is associated with juvenile onset psoriasis and psoriatic arthritis. J Invest Dermatol. 1997;109(4):562-565.

21. Reich K, Huffmeier U, Konig IR, et al. TNF polymorphisms in psoriasis: association of psoriatic arthritis with the promoter polymorphism TNF*-857 independent of the PSORS1 risk allele. Arthritis Rheum. 2007;56(6):2056-2064.

22. Gelfand JM, Neimann AL, Shin DB, Wang X, Margolis DJ, Troxel AB. Risk of myocardial infarction in patients with psoriasis. JAMA. 2006;296(14):1735-1741.

23. Henseler T, Christophers E. Disease concomitance in psoriasis. $J$ Am Acad Dermatol. 1995;32(6):982-986.

24. Neimann AL, Shin DB, Wang X, Margolis DJ, Troxel AB, Gelfand JM. Prevalence of cardiovascular risk factors in patients with psoriasis. J Am Acad Dermatol. 2006;55(5):829-835.

25. Ravindran V, Scott DL, Choy EH. A systematic review and metaanalysis of efficacy and toxicity of disease modifying anti-rheumatic drugs and biological agents for psoriatic arthritis. Ann Rheum Dis. 2008;67(6):855-859.

26. Sommer DM, Jenisch S, Suchan M, Christophers E, Weichenthal M. Increased prevalence of the metabolic syndrome in patients with moderate to severe psoriasis. Arch Dermatol Res. 2006;298(7): 321-328.

27. Fitzgerald O, Winchester R. Psoriatic arthritis: from pathogenesis to therapy. Arthritis Res Ther. 2009;11(1):214

28. Radtke MA, Reich K, Blome C, Rustenbach S, Augustin M. Prevalence and clinical features of psoriatic arthritis and joint complaints in 2009 patients with psoriasis: results of a German national survey. J Eur Acad Dermatol Venereol. 2009;23(6):683-691.

29. Reich K, Kruger K, Mossner R, Augustin M. Epidemiology and clinical pattern of psoriatic arthritis in Germany: a prospective interdisciplinary epidemiological study of 1511 patients with plaque-type psoriasis. Br J Dermatol. 2009;160(5):1040-1047.

30. Biomarkers Definitions Working Group. Biomarkers and surrogate endpoints: preferred definitions and conceptual framework. Clin Pharmacol Ther. 2001;69(3):89-95.

31. Feldman SR, Krueger GG. Psoriasis assessment tools in clinical trials. Ann Rheum Dis. 2005;64 Suppl 2:ii65-ii68. 
32. Langley RG, Ellis CN. Evaluating psoriasis with Psoriasis Area and Severity Index, Psoriasis Global Assessment, and Lattice System Physician's Global Assessment. J Am Acad Dermatol. 2004;51(4): 563-569.

33. Donetti E, Gualerzi A, Ricceri F, Pescitelli L, Bedoni M, Prignano F. Etanercept restores a differentiated keratinocyte phenotype in psoriatic human skin: a morphological study. Exp Dermatol. 2012;21(7): $549-551$.

34. Mommers JM, van Rossum MM, van Erp PE, van De Kerkhof PC. Changes in keratin 6 and keratin 10 (co-)expression in lesional and symptomless skin of spreading psoriasis. Dermatology. 2000;201(1): $15-20$.

35. Rashmi R, Rao KS, Basavaraj KH. A comprehensive review of biomarkers in psoriasis. Clin Exp Dermatol. 2009;34(6):658-663.

36. Krueger JG, Wolfe JT, Nabeya RT, et al. Successful ultraviolet B treatment of psoriasis is accompanied by a reversal of keratinocyte pathology and by selective depletion of intraepidermal T cells. $J$ Exp Med. 1995;182(6):2057-2068.

37. Bhawan J, Bansal C, Whren K, Schwertschlag U. K16 expression in uninvolved psoriatic skin: a possible marker of pre-clinical psoriasis. J Cutan Pathol. 2004;31(7):471-476.

38. Coimbra S, Oliveira H, Reis F, et al. C-reactive protein and leucocyte activation in psoriasis vulgaris according to severity and therapy. J Eur Acad Dermatol Venereol. 2010;24(7):789-796.

39. Rocha-Pereira P, Santos-Silva A, Rebelo I, Figueiredo A, Quintanilha A, Teixeira $\mathrm{F}$. The inflammatory response in mild and in severe psoriasis. Br J Dermatol. 2004;150(5):917-928.

40. Boehncke S, Fichtlscherer S, Salgo R, et al. Systemic therapy of plaque-type psoriasis ameliorates endothelial cell function: results of a prospective longitudinal pilot trial. Arch Dermatol Res. 2011;303(6):381-388.

41. Ridker PM, Morrow DA. C-reactive protein, inflammation, and coronary risk. Cardiol Clin. 2003;21(3):315-325.

42. Strober B, Teller C, Yamauchi P, et al. Effects of etanercept on C-reactive protein levels in psoriasis and psoriatic arthritis. $\mathrm{Br} J$ Dermatol. 2008;159(2):322-330.

43. Kanelleas A, Liapi C, Katoulis A, et al. The role of inflammatory markers in assessing disease severity and response to treatment in patients with psoriasis treated with etanercept. Clin Exp Dermatol. 2011;36(8):845-850.

44. Bhushan M, McLaughlin B, Weiss JB, Griffiths CE. Levels of endothelial cell stimulating angiogenesis factor and vascular endothelial growth factor are elevated in psoriasis. Br J Dermatol. 1999;141(6): 1054-1060.

45. Nielsen HJ, Christensen IJ, Svendsen MN, et al. Elevated plasma levels of vascular endothelial growth factor and plasminogen activator inhibitor-1 decrease during improvement of psoriasis. Inflamm Res. 2002;51(11):563-567.

46. Flisiak I, Zaniewski P, Rogalska-Taranta M, Chodynicka B. Effect of psoriasis therapy on VEGF and its soluble receptors serum concentrations. J Eur Acad Dermatol Venereol. 2011;26(3):302-307.

47. de Jongh GJ, Zeeuwen PL, Kucharekova M, et al. High expression levels of keratinocyte antimicrobial proteins in psoriasis compared with atopic dermatitis. J Invest Dermatol. 2005;125(6):1163-1173.

48. Niyonsaba F, Ogawa H, Nagaoka I. Human beta-defensin-2 functions as a chemotactic agent for tumour necrosis factor-alpha-treated human neutrophils. Immunology. 2004;111(3):273-281.

49. Yang D, Chertov O, Bykovskaia SN, et al. Beta-defensins: linking innate and adaptive immunity through dendritic and T cell CCR6. Science. 1999;286(5439):525-528

50. Jansen PA, Rodijk-Olthuis D, Hollox EJ, et al. Beta-defensin-2 protein is a serum biomarker for disease activity in psoriasis and reaches biologically relevant concentrations in lesional skin. PLoS One. 2009;4(3):e4725.

51. Broome AM, Ryan D, Eckert RL. S100 protein subcellular localization during epidermal differentiation and psoriasis. J Histochem Cytochem. 2003;51(5):675-685.
52. Madsen P, Rasmussen HH, Leffers H, et al. Molecular cloning, occurrence, and expression of a novel partially secreted protein "psoriasin" that is highly up-regulated in psoriatic skin. $J$ Invest Dermatol. 1991;97(4):701-712.

53. Caproni M, Antiga E, Melani L, Volpi W, Del Bianco E, Fabbri P. Serum levels of IL-17 and IL-22 are reduced by etanercept, but not by acitretin, in patients with psoriasis: a randomized-controlled trial. J Clin Immunol. 2009;29(2):210-214.

54. Kerkhoff C, Sorg C, Tandon NN, Nacken W. Interaction of S100A8/ S100A9-arachidonic acid complexes with the scavenger receptor CD36 may facilitate fatty acid uptake by endothelial cells. Biochemistry. 2001;40(1):241-248.

55. Teigelkamp S, Bhardwaj RS, Roth J, Meinardus-Hager G, Karas M, Sorg C. Calcium-dependent complex assembly of the myeloic differentiation proteins MRP-8 and MRP-14. J Biol Chem. 1991; 266(20):13462-13467.

56. Thorey IS, Roth J, Regenbogen J, et al. The Ca2+-binding proteins S100A8 and S100A9 are encoded by novel injury-regulated genes. J Biol Chem. 2001;276(38):35818-35825.

57. Benoit S, Toksoy A, Ahlmann M, et al. Elevated serum levels of calcium-binding $\mathrm{S} 100$ proteins $\mathrm{A} 8$ and $\mathrm{A} 9$ reflect disease activity and abnormal differentiation of keratinocytes in psoriasis. $\mathrm{Br} J$ Dermatol. 2006;155(1):62-66.

58. Anderson KS, Wong J, Polyak K, Aronzon D, Enerback C. Detection of psoriasin/S100A7 in the sera of patients with psoriasis. Br J Dermatol. 2009;160(2):325-332.

59. Arican O, Aral M, Sasmaz S, Ciragil P. Serum levels of TNF-alpha, IFN-gamma, IL-6, IL-8, IL-12, IL-17, and IL-18 in patients with active psoriasis and correlation with disease severity. Mediators Inflamm. 2005;2005(5):273-279.

60. Kagami S, Rizzo HL, Lee JJ, Koguchi Y, Blauvelt A. Circulating Th17, Th22, and Th1 cells are increased in psoriasis. J Invest Dermatol. 2010;130(5):1373-1383.

61. Otkjaer K, Kragballe K, Funding AT, et al. The dynamics of gene expression of interleukin-19 and interleukin-20 and their receptors in psoriasis. Br J Dermatol. 2005;153(5):911-918.

62. Romer J, Hasselager E, Norby PL, Steiniche T, Thorn Clausen J, Kragballe K. Epidermal overexpression of interleukin-19 and -20 mRNA in psoriatic skin disappears after short-term treatment with cyclosporine A or calcipotriol. J Invest Dermatol. 2003;121(6):1306-1311.

63. Wang F, Lee E, Lowes MA, et al. Prominent production of IL-20 by CD68+/CD11c+ myeloid-derived cells in psoriasis: gene regulation and cellular effects. J Invest Dermatol. 2006;126(7):1590-1599.

64. Duan H, Koga T, Kohda F, Hara H, Urabe K, Furue M. Interleukin8-positive neutrophils in psoriasis. J Dermatol Sci. 2001;26(2): 119-124.

65. Ozawa M, Terui T, Tagami H. Localization of IL-8 and complement components in lesional skin of psoriasis vulgaris and pustulosis palmaris et plantaris. Dermatology. 2005;211(3):249-255.

66. Pietrzak AT, Zalewska A, Chodorowska G, et al. Cytokines and anticytokines in psoriasis. Clin Chim Acta. 2008;394(1-2):7-21.

67. Toruniowa B, Krasowska D, Koziol M, Ksiazek A, Pietrzak A. Serum levels of IL-6 in mycosis fungoides, psoriasis, and lichen planus. Ann NY Acad Sci. 1995;762(1):432-434.

68. Zalewska A, Glowacka E, Wyczolkowska J, Tchorzewski H, Narbutt J, Sysa-Jedrzejowska A. Interleukin 6 and 8 levels in plasma and fibroblast cultures in psoriasis. Mediators Inflamm. 2006;2006(1):81767.

69. Flisiak I, Klepacki A, Chodynicka B. Plasma and scales levels of interleukin 18 in comparison with other possible clinical and laboratory biomarkers of psoriasis activity. Biomarkers. 2006;11(2):194-200.

70. Flisiak I, Zaniewski P, Chodynicka B. Plasma TGF-beta1, TIMP-1, MMP-1 and IL-18 as a combined biomarker of psoriasis activity. Biomarkers. 2008;13(5):549-556.

71. Seung NR, Park EJ, Kim CW, et al. Comparison of expression of heatshock protein 60, Toll-like receptors 2 and 4, and T-cell receptor gamma delta in plaque and guttate psoriasis. J Cutan Pathol. 2007;34(12): 903-911. 
72. Wright V. Psoriatic arthritis; a comparative study of rheumatoid arthritis, psoriasis, and arthritis associated with psoriasis. Ann Rheum Dis. 1959;80(1):27-35.

73. Taylor W, Gladman D, Helliwell P, Marchesoni A, Mease P, Mielants H. Classification criteria for psoriatic arthritis: development of new criteria from a large international study. Arthritis Rheum. 2006;54(8):2665-2673.

74. Chandran V, Gladman DD. Update on biomarkers in psoriatic arthritis. Curr Rheumatol Rep. 2010;12(4):288-294.

75. de Vlam K, Gottlieb AB, Fitzgerald O. Biological biomarkers in psoriatic disease. A review. J Rheumatol. 2008;35(7):1443-1448.

76. Tam LS, Tomlinson B, Chu TT, et al. Cardiovascular risk profile of patients with psoriatic arthritis compared with controls - the role of inflammation. Rheumatology (Oxford). 2008;47(5):718-723.

77. Gladman DD, Farewell VT, Nadeau C. Clinical indicators of progression in psoriatic arthritis: multivariate relative risk model. J Rheumatol. 1995;22(4):675-679.

78. Van den Bosch F, Manger B, Goupille P, et al. Effectiveness of adalimumab in treating patients with active psoriatic arthritis and predictors of good clinical responses for arthritis, skin and nail lesions. Ann Rheum Dis. 2010;69(2):394-399.

79. Elkayam O, Yaron I, Shirazi I, Yaron M, Caspi D. Serum levels of IL-10, IL-6, IL-1 ra, and sIL-2R in patients with psoriatic arthritis. Rheumatol Int. 2000;19(3):101-105.

80. Alenius GM, Eriksson C, Rantapaa Dahlqvist S. Interleukin-6 and soluble interleukin-2 receptor alpha-markers of inflammation in patients with psoriatic arthritis? Clin Exp Rheumatol. 2009;27(1):120-123.

81. Szodoray P, Alex P, Chappell-Woodward CM, et al. Circulating cytokines in Norwegian patients with psoriatic arthritis determined by a multiplex cytokine array system. Rheumatology (Oxford). 2007;46(3): 417-425.

82. Chandran V, Cook RJ, Edwin J, et al. Soluble biomarkers differentiate patients with psoriatic arthritis from those with psoriasis without arthritis. Rheumatology (Oxford). 2010;49(7):1399-1405.

83. Dalbeth N, Pool B, Smith T, et al. Circulating mediators of bone remodeling in psoriatic arthritis: implications for disordered osteoclastogenesis and bone erosion. Arthritis Res Ther. 2010;12(4): R164.

84. Anandarajah AP, Schwarz EM, Totterman S, et al. The effect of etanercept on osteoclast precursor frequency and enhancing bone marrow oedema in patients with psoriatic arthritis. Ann Rheum Dis. 2008;67(3):296-301.

85. Ritchlin CT, Haas-Smith SA, Li P, Hicks DG, Schwarz EM. Mechanisms of TNF-alpha- and RANKL-mediated osteoclastogenesis and bone resorption in psoriatic arthritis. J Clin Invest. 2003;111(6):821-831.

86. Fiocco U, Sfriso P, Oliviero F, et al. Synovial effusion and synovial fluid biomarkers in psoriatic arthritis to assess intraarticular tumor necrosis factor-alpha blockade in the knee joint. Arthritis Res Ther. 2010;12(4):R148.

87. van Kuijk AW, Reinders-Blankert P, Smeets TJ, Dijkmans BA, Tak PP. Detailed analysis of the cell infiltrate and the expression of mediators of synovial inflammation and joint destruction in the synovium of patients with psoriatic arthritis: implications for treatment. Ann Rheum Dis. 2006;65(12):1551-1557.

88. Conigliaro P, Scrivo R, Valesini G, Perricone R. Emerging role for NK cells in the pathogenesis of inflammatory arthropathies. Autoimmun Rev. 2011;10(10):577-581.

89. Pollock RA, Pellett FJ, Chandran V, Gladman DD. Expression patterns of natural killer receptor genes in inflamed joints and peripheral blood of patients with psoriatic arthritis. Tissue Antigens. 2011;78(5):345-347.

90. Zhu K, Yin X, Tang X, Zhang F, Yang S, Zhang X. Meta-analysis of NOD2/CARD15 polymorphisms with psoriasis and psoriatic arthritis. Rheumatol Int. 2012;32(7):1893-1900.

91. Bowes J, Eyre S, Flynn E, et al. Evidence to support IL-13 as a risk locus for psoriatic arthritis but not psoriasis vulgaris. Ann Rheum Dis. 2011;70(6):1016-1019.
92. Duffin KC, Freeny IC, Schrodi SJ, et al. Association between IL13 polymorphisms and psoriatic arthritis is modified by smoking. J Invest Dermatol. 2009;129(12):2777-2783.

93. Spadaro A, Rinaldi T, Riccieri V, Valesini G, Taccari E. Interleukin 13 in synovial fluid and serum of patients with psoriatic arthritis. Ann Rheum Dis. 2002;61(2):174-176.

94. Love TJ, Gudjonsson JE, Valdimarsson H, Gudbjornsson B. Psoriatic arthritis and onycholysis - results from the cross-sectional Reykjavik Psoriatic Arthritis Study. J Rheumatol. 2012;39(7):1441-1444.

95. Williamson L, Dalbeth N, Dockerty JL, Gee BC, Weatherall R, Wordsworth BP. Extended report: nail disease in psoriatic arthritis clinically important, potentially treatable and often overlooked. Rheumatology (Oxford). 2004;43(6):790-794.

96. Mehta NN, Azfar RS, Shin DB, Neimann AL, Troxel AB, Gelfand JM. Patients with severe psoriasis are at increased risk of cardiovascular mortality: cohort study using the General Practice Research Database. Eur Heart J. 2010;31(8):1000-1006.

97. Stern RS. Psoriasis is not a useful independent risk factor for cardiovascular disease. J Invest Dermatol. 2010;130(4):917-919.

98. Wakkee M, Herings RM, Nijsten T. Psoriasis may not be an independent risk factor for acute ischemic heart disease hospitalizations: results of a large population-based Dutch cohort. J Invest Dermatol. 2010;130(4):962-967.

99. Rietzschel E, De Buyzere M. High-sensitive C-reactive protein: universal prognostic and causative biomarker in heart disease? Biomark Med. 2012;6(1):19-34.

100. Ouchi N, Parker JL, Lugus JJ, Walsh K. Adipokines in inflammation and metabolic disease. Nat Rev Immunol. 2011;11(2):85-97.

101. Boehncke S, Thaci D, Beschmann H, et al. Psoriasis patients show signs of insulin resistance. Br J Dermatol. 2007;157(6): 1249-1251.

102. Johnston A, Arnadottir S, Gudjonsson JE, et al. Obesity in psoriasis: leptin and resistin as mediators of cutaneous inflammation. $\mathrm{Br} \mathrm{J}$ Dermatol. 2008;159(2):342-350.

103. Cerman AA, Bozkurt S, Sav A, Tulunay A, Elbasi MO, Ergun T. Serum leptin levels, skin leptin and leptin receptor expression in psoriasis. Br J Dermatol. 2008;159(4):820-826.

104. Leonardi C, Matheson R, Zachariae C, et al. Anti-interleukin17 monoclonal antibody ixekizumab in chronic plaque psoriasis. N Engl J Med. 2012;366(13):1190-1199.

105. Menter A, Griffiths CE. Current and future management of psoriasis. Lancet. 2007;370(9583):272-284.

106. Papp KA, Leonardi C, Menter A, et al. Brodalumab, an anti-interleukin17-receptor antibody for psoriasis. N Engl J Med. 2012;366(13): 1181-1189.

107. Mease PJ, Goffe BS, Metz J, VanderStoep A, Finck B, Burge DJ. Etanercept in the treatment of psoriatic arthritis and psoriasis: a randomised trial. Lancet. 2000;356(9227):385-390.

108. Antoni C, Manger B. Infliximab for psoriasis and psoriatic arthritis. Clin Exp Rheumatol. 2002;20(6 Suppl 28):S122-S125.

109. Gratacos J, Casado E, Real J, Torre-Alonso JC. Prediction of major clinical response (ACR50) to infliximab in psoriatic arthritis refractory to methotrexate. Ann Rheum Dis. 2007;66(4):493-497.

110. Lebwohl M, Leonardi C, Griffiths CE, et al. Long-term safety experience of ustekinumab in patients with moderate-to-severe psoriasis (Part I of II): results from analyses of general safety parameters from pooled Phase 2 and 3 clinical trials. J Am Acad Dermatol. 2011;66(5):731-741.

111. Boehncke S, Salgo R, Garbaraviciene J, et al. Effective continuous systemic therapy of severe plaque-type psoriasis is accompanied by amelioration of biomarkers of cardiovascular risk: results of a prospective longitudinal observational study. J Eur Acad Dermatol Venereol. 2011;25(10):1187-1193.

112. Zaba LC, Suarez-Farinas M, Fuentes-Duculan J, et al. Effective treatment of psoriasis with etanercept is linked to suppression of IL-17 signaling, not immediate response TNF genes. J Allergy Clin Immunol. 2009;124(5):1022-1010. 
113. Suarez-Farinas M, Shah KR, Haider AS, Krueger JG, Lowes MA. Personalized medicine in psoriasis: developing a genomic classifier to predict histological response to Alefacept. BMC Dermatol. 2010;10:1.

114. Swindell WR, Xing X, Stuart PE, et al. Heterogeneity of inflammatory and cytokine networks in chronic plaque psoriasis. PLoS One. 2012;7(3):e34594.

115. Taylor PC, Peters AM, Paleolog E, et al. Reduction of chemokine levels and leukocyte traffic to joints by tumor necrosis factor alpha blockade in patients with rheumatoid arthritis. Arthritis Rheum. 2000;43(1):38-47.
116. Caprioli F, Bosè F, Rossi R, et al. Reduction of $\mathrm{CD} 68^{+}$macrophages and decreased IL-17 expression in intestinal mucosa of patients with inflammatory bowel disease strongly correlate with endoscopic response and mucosal healing following infliximab therapy. Inflamm Bowel Dis. 2012. [In press]

\section{Publish your work in this journal}

Psoriasis: Targets and Therapy is international, peer-reviewed, open access journal focusing on psoriasis, nail psoriasis, psoriatic arthritis and related conditions, identification of therapeutic targets and the optimal use of integrated treatment interventions to achieve improved outcomes

\section{Dovepress}

and quality of life. Visit http://www.dovepress.com/testimonials.php to read real quotes from published authors. 